a machine-learning algorithm to design the molecule and subsequently optimize its selfassembling behaviour.

Previous studies have explored ways to combine benefits from peptide amphiphiles and $\mathrm{ELPs}^{8,9}$. However, by incorporating PTM as a programmable step in the synthesis process, Chilkoti and co-workers do so while creating more complex molecular structures that would otherwise be too difficult to synthesize. Furthermore, the possibility to have distinct segments within the same molecule that assemble through different mechanisms permits the generation of hierarchical supramolecular structures and the emergence of new properties with temporal control. Consequently, these materials may find great use in applications that would benefit from having temporally controlled or dynamic properties such as in injectable biomaterials, responsive tissue engineered scaffolds, or drug delivery vehicles.

Beyond demonstrating how PTM can be used as a routine step for engineering complex protein-based materials, the study makes important additional contributions. First, the reported one-pot synthesis opens the possibility to engineer complex macromolecules by recombinant expression using more than just the 20 canonical amino acids. Second, the approach introduces a methodology that facilitates the manipulation of macromolecules with temporal and spatial control during the synthesis process. This is a critical strategy that nature uses to generate the unique structure-function relationship found in biological structures and tissues, and addresses a major challenge in soft material science. Third, the study provides insight into the role of molecular order and disorder in protein structure and activity. There is increasing evidence that both ordered and disordered regions of proteins are critical to the functionality of a large part of the proteome $^{10}$. The technique presented by Chilkoti and co-workers not only provides a new tool to study this order-disorder interplay by manipulating each segment separately but also offers the possibility to incorporate protein order-disorder synergies in the design of new materials.

Overall, this study presents an exciting methodology that enhances our capacity to design and engineer materials with proteins while tapping into their true potential.
This approach could take us a step closer to recreating the remarkable functionality of biological materials and opens new opportunities for exploration and discovery within the broad materials community.

Alvaro Mata

School of Engineering and Materials Science, Queen Mary University of London, Mile End Road, London, UK.

Twitter:@Mata_Lab

e-mail:a.mata@qmul.ac.uk

Published online: 19 April 2018

https://doi.org/10.1038/s41557-018-0049-0

References

1. Brodin, J. D. et al. Nat. Chem 4, 375-382 (2012).

King, N. P. et al. Nature 510, 103-108 (2014)

3. Mozhdehi, D. et al. Nat. Chem. http://doi.org/s41557-018-0005-Z (2018).

4. Pinkas, D. M., Ding, S., Raines, R. T. \& Barron, A. E. ACS Chem. Biol. 6, 320-324 (2011).

5. Lim, S. et al. Biotechnol. Prog. 27, 1390-1396 (2011).

6. Hendricks, M. P., Sato, K., Palmer, L. C. \& Stupp, S. I. Acc. Chem. Res. 50, 2440-2448 (2017)

7. Urry, D. W. J. Phys. Chem. B 101, 11007-11028 (1997).

8. Aluri, S., Pastuszka, M. K., Moses, A. S. \& MacKay, J. A. Biomacromolecules 13, 2645-2654 (2012).

9. Inostroza-Brito, K. E. et al. Nat. Chem 7 , 897-904 (2015).

10. van der Lee, R. et al. Chem. Rev. 114, 6589-6631 (2014).

\title{
CROSS-COUPLING
}

\section{All about that base}

Carbon-nitrogen bonds are important linkages that are widespread throughout natural products, organic intermediates and pharmaceuticals and thus their formation has been an active area of research for over 100 years. Typical reaction conditions feature inorganic bases; however, they lead to a variety of issues, such as problems with reproducibility and the need for higher temperatures, increased catalyst loading, or phase-transfer agents. Looking to address some of these concerns and expand the currently limited use of organic bases, Stephen Buchwald and co-workers (J. Am. Chem. Soc. https://doi.org/cm35; 2018) from the Massachusetts Institute of Technology have developed conditions that use 1,8-diazabicyclo[5.4.0] undec-7-ene (DBU) as a base for $\mathrm{C}-\mathrm{N}$ cross-coupling (pictured, top).

The newly developed conditions featuring an air-stable palladium precatalyst $\mathrm{COD}(\mathrm{AlPhos}-\mathrm{Pd})_{2}$ - have been shown to work well for a variety of coupling partners, giving high yields even for basesensitive substrates that are troublesome to couple under previously studied conditions. Fluoroalkylamines, for example, have been reported to undergo base-promoted
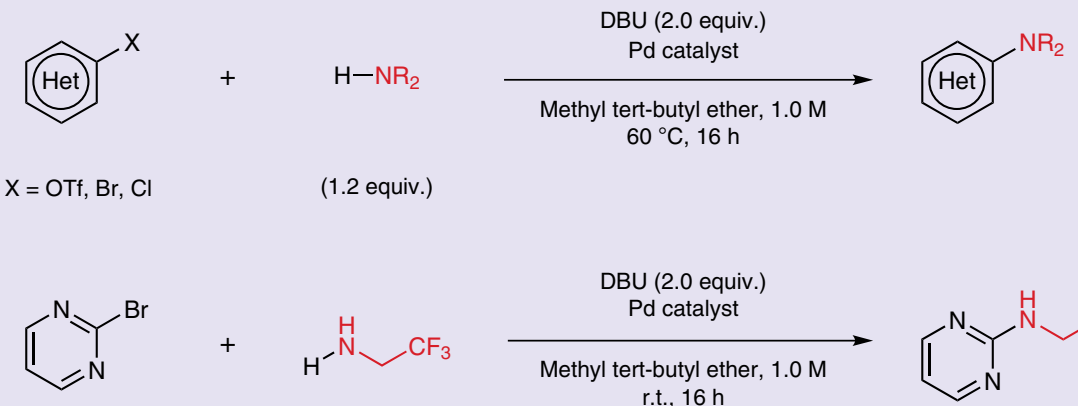

decomposition, but this is not the case when coupled using DBU at room temperature (pictured, bottom). Coupling substrates with alkyl halides has also been shown to work well, allowing the $\mathrm{C}-\mathrm{N}$ coupled product to undergo further synthetic expansion.

In addition to scope, Buchwald and co-workers also investigated the catalytic palladium complex, looking at how ligand size affects the electrophilicity of the palladium atom, and how this in turn affects the base strength required for efficient coupling; this enabled them to explain why AlPhos works well as a ligand in this system. They measured the magnitude of the trans phosphorus-

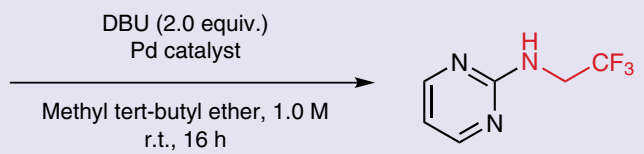

nitrogen coupling constants in several amine-bound palladium complexes featuring ligands of varying size, and out of the ligands investigated, AlPhos led to the smallest coupling constant. This indicates that the complex featuring AlPhos has the most cationic palladium atom and therefore generates a bound amine that is more easily deprotonated - thus leading to efficient coupling even with a weak organic amine base such as DBU.

Katherine Geogheghan

Published online: 19 April 2018

https://doi.org/10.1038/s41557-018-0057-0 